\title{
Sequence Analysis of the Phytoene Synthase Gene (PSY2) in Potato (Solanum Tuberosum)
}

\author{
Jie Ma, Xianzhi Wu, Rui Wu, Chunyan Chen, Yi Lu, Chaohai Wang and \\ Ping Zhou ${ }^{\text {a, }}$ * \\ Bijie Institute of Agricultural Science, Bijie 551700, China. \\ *,a18748560@qq.com
}

Keywords: Potato, carotenoids, phytoene synthase (PSY2), sequence analysis.

\begin{abstract}
Phytoene Synthase (PSY) is an important enzyme in carotenoid biosynthesis. Here, the Solanum tuberosum PSY2 (StPSY2) gene sequences were obtained from Spud DB database, and preformed for sequence analysis. The StPSY2 gene mapped to chromosomes 2, and contains an open reading frame of $1,317 \mathrm{bp}$ that encodes a 438 -amino acid protein with a calculated molecular mass of $49.42 \mathrm{kD}$ and an isoelectric point (pI) of 8.76. Subcellular localization predicted the StPSY2 gene was in the chloroplast. The conserved domain of the StPSY2 protein is PLN02632. The StPSY2 protein is most closely related to Solanum lycopersicum. The findings of the present study provide a molecular basis for the elucidation of PSY2 gene function in potato.
\end{abstract}

\section{Introduction}

Potato (Solanum tuberosum) is ranked as the third most important food crop in the world. Potato is not only of importance as a food crop, and also one of the major crops grown for starch production [1]. It yields a high-energy output per hectare, and is a rich source of nutrients, including carbohydrates, and carotenoids [1-2].

Carotenoids, which are synthesized in various photosynthetic and non-photosynthetic organisms, including algae, plants, and some bacteria and fungi, are a class of 40-carbon hydrocarbon compounds derived from a terpenoid precursor [3-5]. Carotenoids represent a diverse group of pigments that contribute to the red, orange, and yellow colors of various horticultural plants, contributing to their economic value [5-6]. Carotenoids participate in various plant physiological processes, including growth, development, and responses to multiple environmental factors. In green tissues, these act as accessory pigments for the assembly of photosystems and light-harvesting antenna complexes and also have photoprotective functions during photosynthesis $[3,5]$. In non-green tissues, these confer distinct colors to specialized plastids known as chromoplasts, which contribute to the sundry colors in plants $[4,6]$. In addition, carotenoids are the precursors of numerous volatile flavor compounds and phytohormones such as abscisic acid (ABA) and strigolactones [4]. Carotenoids are also essential compounds of human diets, primarily as a precursor of vitamin A [7]. Moreover, carotenoid intake could also reduce the risk for cancer and cardiovascular disease [8-9].

PSY is a rate-limiting enzyme for the biosynthesis of carotenoids, catalyzing the dimerization of two molecules of GGPP into phytoene [10]. The genes encoding the PSY protein have been isolated in various plant species, including Arabidopsis [11], Citrus sinensis [10], and Brassica Rapa [12]. To date, research studies on PSY in potato are limited. In the present study, the PSY2 gene sequence of potato was obtained from web database, and then sequence analysis of the PSY2 gene was analyzed. The present study aimed to establish the foundation for further studies on the molecular mechanism of PSY in potato. 


\section{Materials and Methods}

\subsection{Sequence Obtention of the StPSY2 Gene.}

The genomic DNA and mRNA sequences of PSY2 gene of potato were downloaded and obtained from The Spud DB database (http://solanaceae.plantbiology.msu.edu), and then used to subsequent bioinformatic analysis.

\subsection{Sequence Analysis of the StPSY2 Gene.}

The amino acid sequence, protein molecular weight, isoelectric point, stability index, and hydrophobicity of the StPSY2 gene were analyzed and predicted by ExPASy (http://web.expasy.org) and NCBI (https://www.ncbi.nlm.nih.gov/). Subcellular localization was predicted by WoLF PSORT (http://www.genscript.com/wolf-psort.html). The conserved domain were predicted by NCBI (https://www.ncbi.nlm.nih.gov/Structure/cdd/wrpsb.cgi). Homology analysis of the PSY proteins was executed in DNAMAN.

\section{Results}

\subsection{Analysis on Genomic Organization.}

The Spud DB database was used to analyze the chromosomal localization and genomic organization of StPSY2. The gene ID in Spud DB database is PGSC0003DMT400043103. The StPSY2 gene was mapped to chromosomes 2 and has 6 exons and 5 introns (Fig. 1).

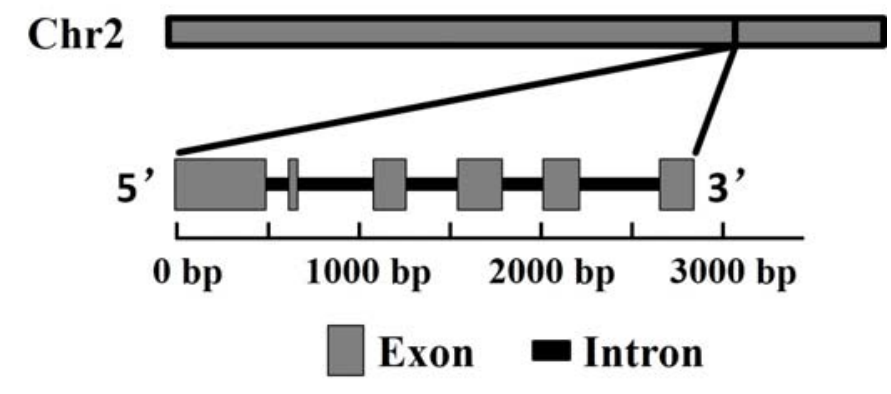

Fig. 1. Chromosomal location and genomic structure of StPSY2.

\subsection{Protein Physical and Chemical Properties Analysis.}

Sequence analysis indicated that the StPSY2 gene contained a 1,317-bp open reading frame (ORF), which encoded a 438-amino acids protein with a calculated molecular mass of $49.42 \mathrm{kD}$ and an isoelectric point ( $\mathrm{pI}$ ) of 8.76. The amino acid types and proportions of the StPSY2 gene was shown in Figure 2, the highest number of amino acid is Leucine (Leu), whereas the lowest number is Histidine (His). Its predicted formula was C2202H3469N611O648S17. Its total average hydrophilicity index was -0.304 , liposoluble index was 84.84 , and instability index in solution was 52.44 .

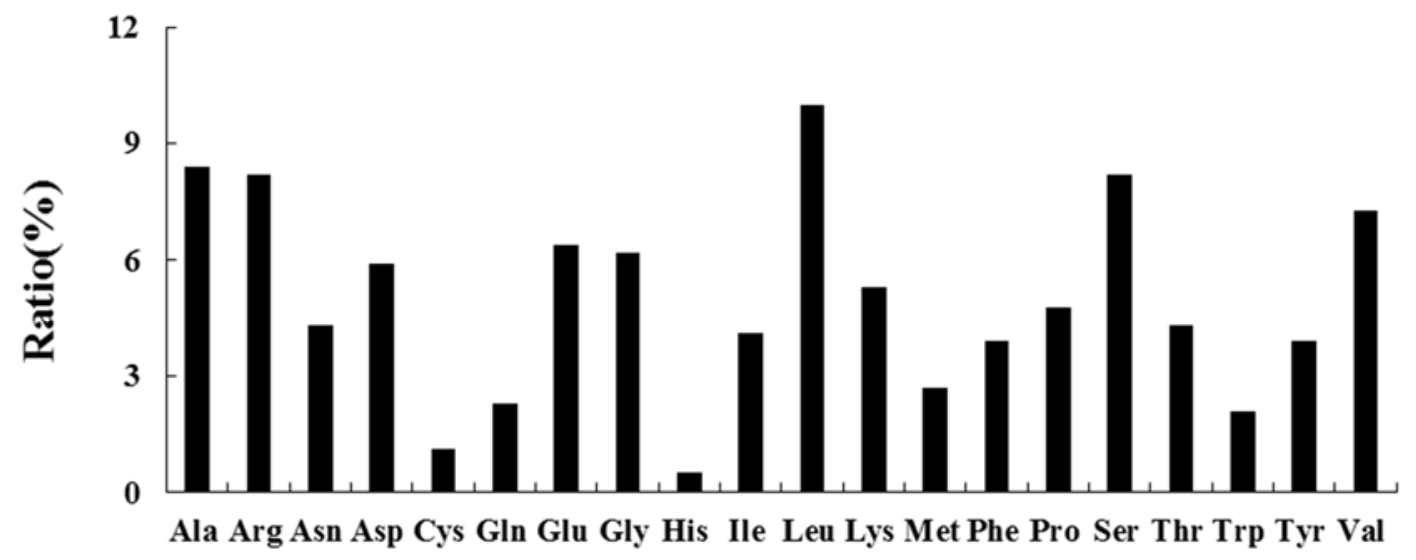

Amino Acid

Fig. 2. Amino acid composition of StPSY2 


\subsection{Subcellular Localization and Conserved Domain Analysis.}

Subcellular localization of the StPSY2 gene was predicted by WoLF PSORT to be in the chloroplast. The analysis using Conserved Domain Database (CDD) demonstrated that the amino acid sequence of the StPSY2 protein has one conserved domain PLN02632 and Isoprenoid_Biosyn_C1 superfamily (Fig. 3).

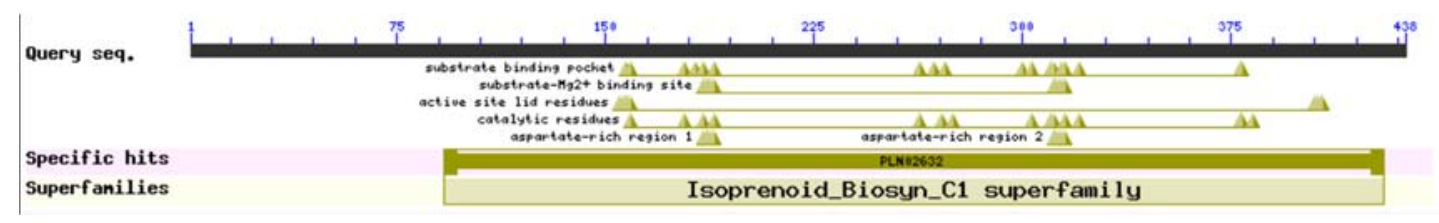

Fig. 3. Conserved domains analysis of StPSY2

\subsection{Homology Analysis.}

Homology analysis demonstrated that the amino acid sequence of the StPSY2 protein shared high homology with those of 18 other higher plant species (Table 1). Table 1 shows that the StPSY2 had the highest identities (>90\%) with several PSY proteins of Solanaceae such as Solanum lycopersicum, Lycium barbarum, Capsicum chinense, and all of the levels of identity were $>75 \%$ with other species cited in our study, indicating that the PSY protein is highly conserved among different species.

Table 1. The homology comparison among amino acid sequences of PSY from plant species

\begin{tabular}{cccc}
\hline Plant species & Protein name & GenBank accession No. & Identity with StPSY2 (\%) \\
\hline Solanum lycopersicum & SIPSY & NP_001234671.1 & 97 \\
Lycium barbarum & LyPSY & AIX87494.1 & 91 \\
Lycium ruthenicum & LrPSY & AIX87518.1 & 91 \\
Nicotiana tabacum & NtPSY & ADZ24219.1 & 90 \\
Capsicum chinense & CcPSY & PHU26731.1 & 91 \\
Capsicum baccatum & CbPSY & PHT56318.1 & 86 \\
Ipomoea batatas & IbPSY & AIY60805.1 & 82 \\
Herrania umbratica & HuPSY & XP_021297941.1 & 74 \\
Prunus mume & PmPSY & NP_001280188.1 & 76 \\
Vitis vinifera & VvPSY & AFP28795.1 & 76 \\
Theobroma cacao & TcPSY & EOY03122.1 & 75 \\
Prunus avium & PaPSY & XP_021823831.1 & 77 \\
Prunus persica & PpPSY & XP_007215407.1 & 77 \\
Hevea brasiliensis & HbPSY & XP_021642840.1 & 77 \\
Camellia sinensis & CsPSY & AJB84620.1 & 78 \\
Manihot esculenta & MePSY & ACY42669.1 & 77 \\
Nicotiana attenuate & NaPSY & OIT34597.1 & 81 \\
Sesamum indicum & SiPSY & XP_011096926.1 & 80 \\
\hline
\end{tabular}

\section{Summary}

The present study analyzed the StPSY2 gene of potato. Overexpression of the CsPSY gene of Citrus sinensis in Hongkong kumquat results in a 2.5-fold increase of phytoene accumulation in transgenic fruits, and lycopene, $\beta$-carotene, and $\beta$-cryptoxanthin were also markedly increased [10]. Previous studies have shown that the PSY protein is relatively conserved in plants [10-11]. The PSY protein of Citrus sinensis is similar to the PSY protein of Citrus unshiu and Cucumis melo, showing $99 \%$ and $82 \%$ homology, respectively [10]. The findings of the present study show that PSY from potato is highly conserved in plants, similar to that observed in earlier reports. The findings of the present study may serve as a foundation for future studies on the functions of StPSY2 in carotenoid metabolism in potato. 


\section{Acknowledgements}

This work was supported by Science and Technology Foundation of Guizhou Province (Qian Ke He Ji Chu [2016]1003; Qian Ke He Ji Chu [2018]1402), The Project of Bijie Experimental Station in National Industry Technical System of Potato (CARS-10-ES19), and Science and Technology Special Fund Project of Central Subsidized Place (Qian Ke He Tiao Zhong Bu Di [2015]4003).

\section{References}

[1]. M. Andersson, H. Turesson, A. Nicolia, A. Fält, M. Samuelsson, and P. Hofvander, Efficient targeted multiallelic mutagenesis in tetraploid potato (Solanum tuberosum) by transient CRISPRCas9 expression in protoplasts, Plant Cell Rep. 36 (2017) 117-128.

[2]. S. Lu, J. Van Eck, X.J. Zhou, A.B. Lopez, D.M. O’Halloran, K.M. Cosman, B.J. Conlin, D.J. Paolillo, D.F. Garvin, J. Vrebalov, L.V. Kochian, H. Küpper, E.D. Earle, J. Cao, L. Li, the cauliflower or gene encodes a dnaJ cysteine-rich domain-containing protein that mediates high levels of beta-carotene accumulation, Plant Cell 18 (2006) 3594-3605.

[3]. L. Pizarro, and C. Stange, Light-dependent regulation of carotenoid biosynthesis in plants, Cien. Inv. Agr. 36 (2009) 143-162.

[4]. L.H. Liu, Z.Y. Shao, M. Zhang, and Q.M. Wang, Regulation of carotenoid metabolism in tomato, Mol. Plant 8 (2015) 28-39.

[5]. N. Nisar, L. Li, S. Lu, N.C. Khin, and B.J. Pogson, Carotenoid metabolism in plants, Mol. Plant 8 (2015) 68-82.

[6]. P.A. Tuan, J.K. Kim, J. Lee, W.T. Park, D.Y. Kwon, Y.B. Kim, H.H. Kim, H.R. Kim, and S.U. Park, Analysis of carotenoid accumulation and expression of carotenoid biosynthesis genes in different organs of Chinese cabbage (Brassica rapa subsp. pekinensis), Excli J. 11 (2012) 508516.

[7]. N.I. Krinsky, and E.J. Johnson, Carotenoid actions and their relation to health and disease, Mol. Aspects. Med. 26 (2005) 459-516.

[8]. S.T. Mayne, Beta-carotene, carotenoids, and disease prevention in humans, FASEB J. 10 (1996) 690-701.

[9]. F. Giovannucci, Tomatoes, tomato-based products, lycopene, and cancer: review of the epidemiologic literature, J. Natl. Cancer Inst. 91 (1999) 317-331.

[10]. J.C. Zhang, N.G. Tao, Q. Xu, W.J. Zhou, H.B. Cao, J. Xu, and X.X. Deng, Functional characterization of Citrus PSY gene in Hongkong kumquat (Fortunella hindsii Swingle), Plant Cell Rep. 28 (2009) 1737-1746.

[11]. P.A. Scolnik, and G.E. Bartley, Nucleotide sequence of an Arabidopsis cDNA for phytoene synthase, Plant Physiol. 104 (1994) 1471-1472.

[12]. P.R. Li, S.J. Zhang, S.F. Zhang, F. Li, H. Zhang, F. Cheng, J. Wu, X.W. Wang, R.F. Sun, Carotenoid biosynthetic genes in Brassica rapa: comparative genomic analysis, phylogenetic analysis, and expression profiling, BMC Genomics 16 (2015) 492. 